\title{
LA ELECTROCOAGULACIÓN COMO UN TRATAMIENTO EFICIENTE PARA LA REMOCIÓN DE METALES PESADOS PRESENTES EN AGUAS RESIDUALES
}

Fecha de recepción: 23 de octubre de 2013 • Fecha de aceptación: 26 de noviembre de 2013

\author{
Electrocoagulation as an efficient treatment for the removal of heavy metals from \\ wastewater \\ Gema Eunice Acosta Niño ${ }^{1}$ Carlos Andrés Coy Barrera, PhD. ${ }^{2}$ Alejandro Bourdón García ${ }^{3}$ Elisabeth Cuervo \\ Lumbaque ${ }^{4}$
}

\section{RESUMEN}

La remoción de metales pesados en aguas residuales por algunos métodos ha preocupado a las autoridades ambientales, debido a los altos costos, además de la generación de residuos secundarios y en algunas ocasiones la poca eficiencia de los procesos empleados. Con el fin de retirar metales pesados en aguas, se han desarrollado estudios en el campo de la electrocoagulación, postulando dicho proceso como alternativa viable para el tratamiento de aguas residuales, ya que éste genera pocos residuos secundarios, es rentable económicamente y tiene altos porcentajes de remoción, cercanos o iguales al cien por ciento. El presente trabajo muestra una recopilación bibliográfica de los últimos años que evidencia el uso e importancia de la electrocoagulación con el fín de remover diferentes metales como: hierro, níquel, cobre, zinc, plomo, cadmio, mercurio y cromo hexavalente, presentes en aguas residuales, para mejorar los parámetros en el proceso de remoción de estos contaminantes ambientales que son tan perjudiciales para la salud.

Palabras clave: Electrocoagulación, aguas residuales, metales pesados.

1 M. Sc. Química, Profesor Asociado, Facultad de Ciencias, Universidad Militar Nueva Granada, Bogotá D.C., Colombia, gema.acosta@unimilitar.edu.co

2 PhD. Química, Profesor Auxiliar, Facultad de Ciencias, Universidad Militar Nueva Granada, Bogotá D.C., Colombia, carlos.coy@unimilitar.edu.co

3 Químico, Investigador externo, Profesor catedrático Universidad Militar Nueva Granada, Bogotá D.C., Colombia, jose.bourdon@unimilitar.edu.co

4 Licenciada en Química, Investigadora externa, Universidad Militar Nueva Granada, Bogotá D.C., Colombia, ecuervolumbaque@gmail.com 


\begin{abstract}
The removal of heavy metals in waste water by some methods has concerned environmental authorities, due to the high costs, in addition to the generation of secondary waste and sometimes the low efficiency of the processes used. In order to remove heavy metals in water, have carried out studies in the field of electrocoagulation postulate that process as a viable wastewater treatment alternative, since it generates little secondary waste, is cost-effective and has high percentages removal, close or equal to $100 \%$. This paper shows a bibliography of recent years demonstrating the use and importance of electrocoagulation in order to remove various metals such as iron, nickel, copper, zinc, lead, cadmium, mercury and hexavalent chromium present in wastewater in order to improve the parameters in the process of removal of these environmental pollutants are so harmful to health.
\end{abstract}

Keywords: electrocoagulation, wastewater, heavy metals.

\title{
INTRODUCCIÓN
}

Los altos índices de contaminación, debido a elevadas concentraciones de metales pesados presentes en agua residuales, producto de actividades industriales, tienen como resultado el deterioro de ríos, lagos y otros cuerpos de agua, que son importantes para cualquier tipo de ecosistema. Al igual, el incremento de la población, la expansión de las zonas urbanas y la creciente industrialización, agravan los impactos adversos sobre el recurso hídrico. Esto lleva a que los residuos líquidos sean objeto de tratamiento antes de ser vertidos a los cuerpos de agua naturales, además, los metales pesados son considerados, entre otros aspectos, inhibidores de procesos biológicos y dañinos para la salud (Domingo, 2008; Daphia, 2002; Soto et al., 2004).

Dentro de las industrias que causan mayor contaminación al agua, la galvanoplastia es una de las principales (Milanez et al., 2005), ya que los residuos generados son potencialmente contaminantes, encontrándose conformados mayoritariamente por metales pesados tales como zinc, níquel, cromo, cobre y cadmio.

Es así como actividades de los diferentes sectores industriales se ven en la obligación de evitar o minimizar los impactos negativos al ambiente (Restrepo et al., 2006), ya sea a través de la implementación de prácticas de prevención de la contaminación o del tratamiento de aguas residuales antes de ser vertidos al medio ambiente (Chávez et al., 2009).

Con el fin de controlar, minimizar y en algunos casos eliminar los impactos ambientales asociados al agua contaminada, en las últimas décadas se han emprendido acciones encaminadas a identificar, cuantificar, evaluar e intervenir aquellas industrias generadoras de contaminantes, especialmente de metales pesados debido a su alto grado de toxicidad, esto, con la formulación y aplicación de estrategias sostenibles de gestión del agua, para dar un uso racional al recurso hídrico y su posterior reutilización, revirtiendo la tendencia de escasez que se ha 
presentado en el último siglo (Holt et al., 2002), lo que lleva a una inminente necesidad por desarrollar nuevas tecnologías con mayor eficiencia y de bajo costo para tratar estas aguas residuales, específicamente en este punto es donde emerge el uso de la técnica de electrocoagulación como una opción eficiente, teniendo como ejemplo el caso del plomo. (Eiband et al., 2014), (Eiband et al., 2014) y (Merzouk et al., 2009).

Dentro de las industrias que causan mayor contaminación al agua, la galvanoplastia es una de las principales (Milanez et al., 2005), ya que los residuos generados son potencialmente contaminantes, encontrándose conformados mayoritariamente por metales pesados tales como zinc, níquel, cromo, cobre y cadmio.

En los últimos años los sistemas de control de contaminación denominados "al final del tubo", han sido utilizados para controlar los desechos generados por los procesos industriales. Sin embargo, estos sistemas están asociados a una cantidad importante de problemas ambientales y altos algunos de operación (Chabalina, 2002), lo cual es poco viable ecológica y económicamente (FUNDES, 2003), causando una ineficiencia al momento de aplicar un tratamiento a las aguas residuales que puedan ser incorporados a los diferentes cuerpos receptores (Morales y Acosta, 2010).

Es así como actividades de los diferentes sectores industriales se ven en la obligación de evitar o minimizar los impactos negativos al ambiente (Restrepo et al, 2006), ya sea a través de la implementación de prácticas de prevención de la contaminación o del tratamiento de aguas residuales antes de ser vertidos al medio ambiente (Chávez et al., 2009).

El tratamiento de aguas contaminadas con metales pesados ha sido analizado rigurosamente con diversas metodologías, que incluyen fenómenos biotecnológicos, de adsorción, de intercambio iónico, de ultrafiltración, ósmosis inversa y precipitación química (Matlock et al., 2002), teniendo en algunos casos la preconcentración de la solución (Hagarová et al., 2013), (Bahadır et al., 2014) y (Xu et al., 2013).

Algunos de estos tratamientos han presentado problemas en su aplicación, ya que tiene limitaciones al momento de retirar estos metales de los lodos resultantes en proceso industriales, específicamente en el proceso de separación de los contaminantes en la fase sólida, así como al implementar un proceso de bioadsorción (Agouborde, 2008). Por otra parte, la microfiltración (Ritchie, 2001), la ultrafiltración y la ósmosis inversa, evidencian poca viabilidad para las pequeñas empresas debido a los altos costos de operación del proceso y al reemplazo de resinas, para el caso del intercambio iónico (Escobar et al, 2006). Es así como la electrocoagulación se comporta como un proceso que emplea los fundamentos de la electroquímica, la coagulación y flotación o precipitación, para eliminar los contaminantes del agua

\section{Algunos de estos tratamientos han presentado problemas en su aplicación, ya que tiene limitaciones al momento de retirar estos metales de los lodos resultantes en proceso industriales, específicamente en el proceso de separación de los contaminantes en la fase sólida, así como al implementar un proceso de bioadsorción (Agouborde, 2008).}




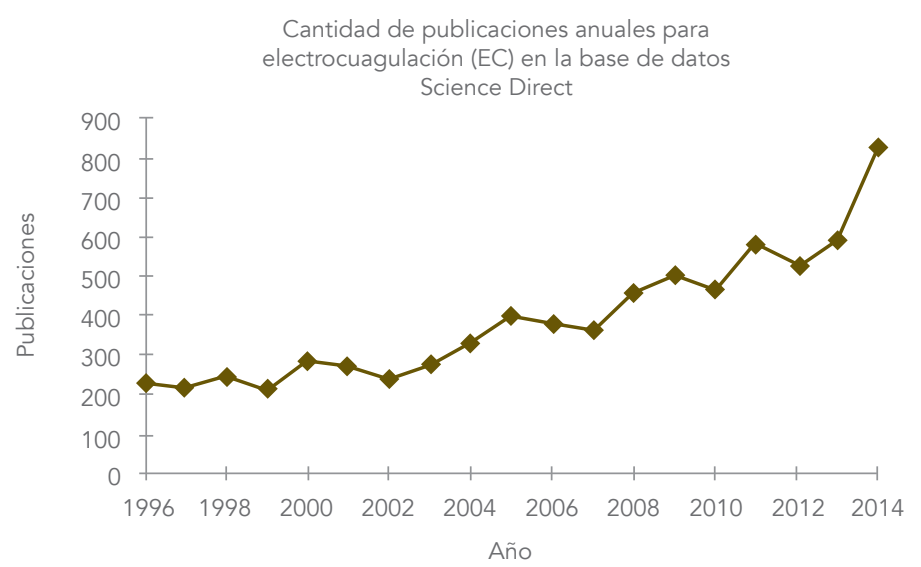

Figura 1. Publicaciones reportadas para electrocoagulación en la base de datos Science Direct, durante los últimos 18 años. El valor para el 2014 se determinó con base en las publicaciones de los primeros 7 meses.

(Holt et al., 2006), con el uso de electrodos (cátodos y ánodos), que por acción de un potencial de energía generan un coagulante, el cual lleva a la coagulación in situ (Eiband et al., 2014), (Eiband et al., 2014). Además, este proceso ha demostrado tener alta eficiencia en la remoción de metales pesados, minimizando la generación de residuos secundarios y por ende el impacto ambiental del proceso. (Merzouk et al., 2009).

La importancia que se da a la investigación en electrocoagulación se ve reflejada en el aumento de las publicaciones durante las dos últimas décadas la cual se ha incrementado en altas proporciones. Se espera que este año la cantidad de publicaciones llegue a un número cercano ochocientos estudios, reportados solamente en la base de datos Science Direct (tomando el ejemplo), como se puede verificar en la figura No.1.

Por su semejanza con la coagulación, es importante resaltar que la electrocoagulación presenta una mayor eficiencia, que se debe a que los cationes generados presentan mayores porcentajes de remoción frente a los provenientes de productos químicos tradicionales como el sulfato de aluminio o hierro. Además, de usar equipos pequeños es posible obtener costos razonables de funcionamiento, posible automatización y menor generación de sales y residuos (Belkacem et al., 2010; Elham et al., 2011). Es posible fabricar los equipos para realizar estos procedimientos a nivel de laboratorio. (Acosta et al., 2013).

El uso de esta clase de tecnología implica utilizar un reactor de electrocoagulación, este equipo se puede considerar como una celda electrolítica cuyos elementos conductores de corriente o electrodos (ánodos o electrodos de trabajo y cátodos o electrodos inertes), son sumergidos en el fluido a tratar, que contiene los metales o sustancias que van a ser retirados (Tchamango et al., 2010) y que es aprovechado como medio electrolítico (Ashraf et al., 2010; Zhao et al., 2011). Los reactores pueden ser en modo discontinuo (Acosta et al., 2013) o continuo (Eiband M. et al 2014).

Para que el reactor funcione, se requiere de una fuente externa de energía eléctrica, que origina las reacciones electroquímicas como consecuencia del flujo de electrones entre los electrodos metálicos y los compuestos presentes en el efluente, generando procesos de reducción en el cátodo (conversión de los protones del agua en hidrógeno), y los de oxidación en el ánodo, que produce iones metálicos y oxígeno, este último proveniente de la hidrólisis del agua (Koboya et al., 2003) (figura 2). Cuando esto ocurre, 


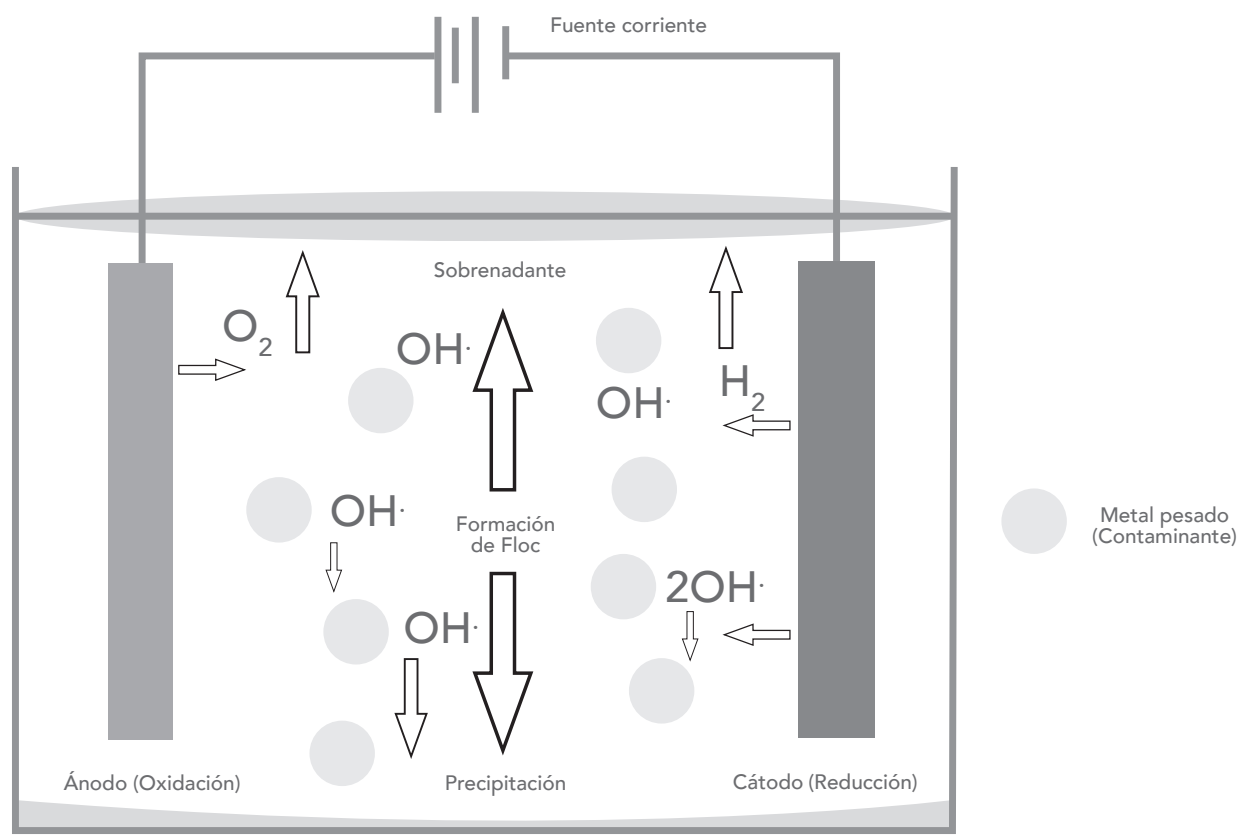

Figura 2. Mecanismo de electrocoagulación en agua contaminada con un metal pesado.

Fuente: (Gráfico realizado por los autores del artículo, Acosta G, Bourdon A, Coy A, Cuervo E)

los contaminantes forman componentes hidrofóbicos que se precipitan o flotan, esto facilitando su remoción por algún método de separación secundario (Ping, 2005; Chavez et al., 2005; Gomes et al., 2007).

Una amplia gama de especies coaguladas e hidróxidos metálicos pueden ser formados a partir de los iones metálicos a un pH apropiado, que conduce a la desestabilización, precipitación y eliminación de contaminantes disueltos tales como metales pesados (Elham et al, 2011).

Dentro de los contaminantes más estudiados se encuentran: arsénico, hierro, níquel, cobre, zinc, plomo, cadmio, mercurio y cromo hexavalente, siendo los electrodos más utilizados en los procesos de electrocoagulación aluminio $\left(\mathrm{Al}^{3+}\right)$ y hierro $\left(\mathrm{Fe}^{2+}\right)$ (Berenguer, 2009; Jing-wei, et al., 2007; Babu et al., 2007; Aristizábal y Bermúdez, 2007), donde se han reportado altos porcentajes de remoción (Aber et al, 2009; Ölmez, 2009).
El presente artículo da a conocer algunos de los parámetros para el proceso de electrocoagulación en el tratamiento de estos contaminantes ambientales presentes en aguas residuales, con costos de remoción menores, citando a modo de ejemplo una solución acuosa con arsénico partiendo de una concentración inicial de 100 ppm, el usar coagulación química y posterior microfiltración calculan un valor de USD\$ 0,36 y al tratar la misma solución por medio de electrocoagulación y posterior microfiltración arroja un valor de USD\$ 0,106 (CESAR CALDERON MOLGORA 2013).

\section{Condiciones óptimas para remoción de contaminantes por electrocoagulación a escala de laboratorio}

En busca de perfeccionar tecnologías más amigables con el ambiente, en los últimos años 
se han desarrollado un sinnúmero de investigaciones para fortalecer el campo de la electroquímica, bajo diferentes parámetros claves como: $\mathrm{pH}$, potencial eléctrico, densidad de corriente y tiempo de electrocoagulación. Para su aplicación el tratamiento de suspensiones, eliminación de compuestos orgánicos en soluciones y aguas residuales provenientes del sector industrial, que contienen metales pesados tales como arsénico, hierro, níquel, cobre, zinc, plomo, cadmio, mercurio y cromo (Gülsün et al., 2009; Holt et al., 2005). La tabla 1 muestra las condiciones óptimas para la remoción de contaminantes por electrocoagulación.

Es importante mencionar que el cromo ha sido ampliamente estudiado (Elham et al., 2011; Yuan et al., 2008) porque posee un amplio uso en la industria metalúrgica y química, sobretodo en la producción de aleaciones, pigmentos, curtido y para la síntesis de agentes oxidantes e inhibidores de corrosión. Además, de ser designado el cromo hexavalente como contaminante por la EPA (Environmental Protection Agency), debido a que tiene una movilidad significativa en el medio ambiente y una toxicidad alta. Las aguas residuales de estas industrias tienen $\mathrm{Cr}^{6+} \circ \mathrm{Cr}^{3+}$, lo cual depende del $\mathrm{pH}$, la presencia de agentes oxidantes o reductores, y la cinética de las reacciones (Bhatti at al., 2009).

De acuerdo a la tabla $N^{\circ} 1$, los métodos de electrocoagulación empleados para el tratamiento de contaminantes industriales muestran un rendimiento de remoción entre el 90 y $100 \%$, donde se obtuvo un buen grado de eficiencia en comparación con otras metodologías biológicas y fotoquímicos (Arango et al., 2007), teniendo en cuenta determinados factores que condicionan la efectividad del reactor como: la producción de burbujas, transporte de masa, distancia, material y geometría de electrodos, densidad de corriente y tipo de conexión (Gilpavas, 2008).

Los resultados obtenidos al usar diferentes tipos de electrodos revelan valores admisibles para el aluminio como ánodo, ya que promueve la formación de hidróxidos y/o polihidróxidos por efecto del $\mathrm{pH}$. Para el hierro, los datos reportados actuando este metal como ánodo son superiores en comparación con los de aluminio, logrando una remoción total del contaminante en corto tiempo (Ivonne Linares-Hernandez 2009). En el caso de sustancias orgánicas en solución acuosa el material depende del parámetro a mejorar, (demanda química de oxígeno, demanda bioquímica de oxígeno, turbidez, etc), teniendo la posibilidad de combinar Al-Al, Al$\mathrm{Fe}$ o Fe-Fe, teniendo como electrodo de sacrificio el hierro cuando se combinan los dos metales (LinaresHernandez, 2009).

Dicha remoción, la complementa la distancia entre los electrodos y su geometría, ya que estos aspectos son claves para el óptimo funcionamiento del reactor (Zhu et al, 2011), debido a que a menor distancia entre placas existe menor resistencia que facilita la rápida formación de floc, esto a su vez dado por una mayor área de los electrodos en contacto con la solución (geometría). Algunos autores usan electrodos de placas paralelas (Acosta et al., 2013) (Mehtap, 2009) mientras que otros se inclinan por electrodos concéntricos (Divagar, 2010).

Al igual que el tipo, distancia y geometría del electrodo en la optimización del sistema de electrocoagulación, la densidad de corriente y el voltaje aplicado también son importantes para lograr altos porcentajes de remoción (Mollah et al, 2004), es así como la densidad de corriente al determinar la producción de oxígeno $\left(\mathrm{O}_{2}\right)$ e hidrógeno $\left(\mathrm{H}_{2}\right)$ gaseoso (Elham et al., 2011), genera una mejor superficie de contacto del coagulante con el contaminante (Gilpavas, 2008).

A su vez, el voltaje aplicado a los diferentes sistemas reportados está directamente relacionado con el tiempo de permanencia del agua residual en el reactor y el desgaste de los electrodos (Bhatti et al., 2009; Heidmann y Calmano, 2008; Merzouk et al., 
Tabla 1. Condiciones óptimas para remoción de contaminantes por electrocoagulación a escala de laboratorio.

\begin{tabular}{|c|c|c|c|c|c|c|}
\hline \multirow[b]{2}{*}{ Variedad } & \multicolumn{4}{|c|}{ Parámetros } & \multirow{2}{*}{$\begin{array}{l}\text { Valores de } \\
\text { remoción }\end{array}$} & \multirow[b]{2}{*}{ Ref. } \\
\hline & $\begin{array}{l}\text { Densidad } \\
\text { corriente }\end{array}$ & $\begin{array}{l}\text { Potencial } \\
\text { eléctrico }\end{array}$ & Tiempo & $\mathrm{pH}$ & & \\
\hline $\begin{array}{l}\text { Aguas residua- } \\
\text { les que contie- } \\
\text { nen } \mathrm{Cu}^{2+}, \mathrm{Zn}^{2+} \\
\text { y } \mathrm{Cr}^{6+}\end{array}$ & $\begin{array}{c}0,8-4.8 \\
\mathrm{~A} / \mathrm{dm}^{2}\end{array}$ & N.R & $\begin{array}{l}20 \text { min } \mathrm{Zn} \text { y } \mathrm{Cu} \\
60 \mathrm{~min} \mathrm{Cr}(\mathrm{VI})\end{array}$ & $4,0-8,0$ & $98 \%$ & $\begin{array}{l}\text { (Adhoum } \\
\text { et al., 2004) }\end{array}$ \\
\hline $\mathrm{Cr}^{3+}$ en agua & $\begin{array}{c}32,52 \\
\mathrm{~mA} / \mathrm{cm}^{2} \\
\text { Electrolito } \\
\text { de apoyo } \\
\mathrm{NaCl}\end{array}$ & N.R & $60 \mathrm{~min}$ & 3,4 & $98 \%$ & $\begin{array}{l}\text { (Golder } \\
\text { et al., 2006) }\end{array}$ \\
\hline $\mathrm{As}^{5+}$ en agua & $30 \mathrm{~mA} / \mathrm{cm}^{2}$ & N.R & $60 \mathrm{~min}$ & 2,4 & $\geq 99,6 \%$ & $\begin{array}{l}\text { (Gomes } \\
\text { et al., 2007) }\end{array}$ \\
\hline $\mathrm{Cr}^{6+}$ en agua & $\begin{array}{c}\text { Corrientes } \leq \\
0,1 \mathrm{~A}\end{array}$ & $0,9 \vee$ & $45 \min$ & N.R & $\begin{array}{c}\text { Remoción } \\
\text { total }\end{array}$ & $\begin{array}{c}\text { (Heidmann y Cal- } \\
\text { man., 2008) }\end{array}$ \\
\hline $\begin{array}{c}\text { Aguas residuales } \\
\text { que contiene } \\
\mathrm{Ni}^{2+}, \mathrm{Cu}^{2+}, \mathrm{Zn}^{2+}\end{array}$ & $0,5 \mathrm{~A} / \mathrm{dm}^{2}$ & N.R & $30 \min$ & 5,4 & $: \geq 98 \%$ & $\begin{array}{l}\text { (Mouedhen } \\
\text { et al., 2008) }\end{array}$ \\
\hline $\mathrm{Cr}^{6+}$ en agua & N.R & $12,8 \mathrm{~V}$ & $24 \min$ & 5,0 & $90,4 \%$ & $\begin{array}{c}\text { (Bhatti } \\
\text { et al., 2009) }\end{array}$ \\
\hline $\mathrm{Cr}^{6+}$ en agua & $\begin{array}{l}200 \mathrm{~A} / \mathrm{m}^{2} \\
\text { Electrodos } \\
\text { de Fe }\end{array}$ & N.R & $30 \mathrm{~min}$ & 9,0 & $\begin{array}{l}\text { Remoción } \\
\text { total }\end{array}$ & $\begin{array}{l}\text { (Zongo } \\
\text { et al., 2009) }\end{array}$ \\
\hline
\end{tabular}

2009; Linares et al, 2009), que también se ven afectados por el nivel de contaminación a tratar y la naturaleza del contaminante (Phalakornkule et al., 2010).

A medida que se realiza el proceso de electrocoagulación se forma una capa de óxido sobre el cátodo, así como el deterioro del ánodo, por la a oxidación, lo cual conduce a la perdida de eficiencia de la celda de electrocoagulación. Estas limitaciones se minimizan con la adición de una placa paralela que contiene el electrodo de sacrificio, suministrando una corriente directa. Aunque muchos autores prefieren el uso de la corriente alterna, puesto que se cree que la activación cíclica retarda los mecanismos normales de ataque del electrodo que experimenta el sistema y así se puede asegurar la vida del electrodo de una manera razonable (Mollah et al., 2001).

Adicionalmente, para lograr mayores y mejores resultados algunos autores optan por la 


\begin{tabular}{|c|c|c|c|c|c|c|}
\hline $\begin{array}{c}\text { Aguas residuales } \\
\text { que contienen } \\
\mathrm{Fe}^{2+}, \\
\mathrm{Ni}^{2+}, \mathrm{Cu}^{2+}, \mathrm{Zn}^{2+} \\
\mathrm{Pb}^{2+} \text { y } \mathrm{Cd}^{2+}\end{array}$ & $\begin{array}{c}11,55 \\
\mathrm{~mA} / \mathrm{cm}^{2}\end{array}$ & $30 \mathrm{~V}$ & $15 \mathrm{~min}$ & 7,6 & $95 \%$ & $\begin{array}{l}\text { (Merzouk } \\
\text { et al., 2009) }\end{array}$ \\
\hline $\mathrm{Cr}^{6+}$ en agua & 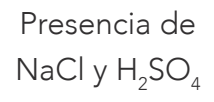 & N.R & $60 \mathrm{~min}$ & 6,5 & $99 \%$ & $\begin{array}{l}\text { (Arroyo } \\
\text { et al., 2009) }\end{array}$ \\
\hline $\begin{array}{c}\text { Agua de río } \\
\text { contaminada } \\
\text { con } \mathrm{Hg}^{2+}\end{array}$ & $\begin{array}{c}2.5-3.125 \mathrm{~A} / \\
d \mathrm{~m}^{2}\end{array}$ & $0.3-0.9 \mathrm{~V}$ & N.R & $3,0-7,0$ & $99.9 \%$ & $\begin{array}{l}\text { (Nanseu-Njiki } \\
\text { et al., 2009) }\end{array}$ \\
\hline \multirow{2}{*}{$\begin{array}{c}\mathrm{Cr}^{6+} \text { en agua } \\
\text { proveniente de } \\
\text { curtiembres }\end{array}$} & $64 \mathrm{~mA} / \mathrm{cm}^{2}$ & \multirow[b]{2}{*}{ N.R } & \multirow[b]{2}{*}{$24 \min$} & \multirow[b]{2}{*}{2,4} & \multirow[b]{2}{*}{$99 \%$} & \multirow[b]{2}{*}{ (Duranteet al., 2011) } \\
\hline & $\begin{array}{c}\text { EDTA como } \\
\text { agente } \\
\text { quelante }\end{array}$ & & & & & \\
\hline $\mathrm{Cr}^{6+}$ en agua & $0,2 \mathrm{~A} / \mathrm{m}^{2}$ & N.R & N.R & 7,0 & $98,2 \%$ & $\begin{array}{l}\text { (Vasudevan } \\
\text { et al., 2011) }\end{array}$ \\
\hline $\begin{array}{c}\text { Aguas residuales } \\
\text { que contiene } \\
\mathrm{Ni}^{2+}, \mathrm{Cu}^{2+}, \mathrm{Cr}^{6+}\end{array}$ & $10 \mathrm{~mA} / \mathrm{cm}^{2}$ & N.R & $20 \mathrm{~min}$ & 3,0 & $\begin{array}{c}\text { Remoción } \\
\text { total }\end{array}$ & $\begin{array}{c}\text { (Akbal y } \\
\text { Camci., 2011) }\end{array}$ \\
\hline Agua con $\mathrm{Hg}^{2+}$ & N.R & $9 \mathrm{~V}$ & $50 \mathrm{~min}$ & 4,5 & $98.5 \%$ & (Chaturvedi, 2013) \\
\hline
\end{tabular}

N.R: Dato no registrado

adición de electrolitos de soporte (Golder et al., 2006; Arroyo et al., 2009) garantizando una mayor presencia de cargas que faciliten la generación del coagulante in-situ.

\section{Combinación de la electrocoagulación con otros métodos}

Algunos estudios sobre la electrocoagulación se han complementado con técnicas como la biosorción (Linares et al., 2007), adsorción (Secula et al., 2012), flotación (Filho et al., 2012) y ultrafiltración (Timmes et al., 2009), para tratar aguas residuales, donde se reportan porcentajes de remoción de contaminantes mayores al $97 \%$. El Cry As son los metales pesados que presentan mayor porcentaje de remoción (Durante et al., 2011; Zhao et al., 2011), alcanzando valores de $99 \%$. Sin embargo, no son relevantes dichos resultados puesto 
que otros estudios que utilizan solamente la técnica de electrocoagulación han dado rendimientos iguales o mayores a los reportados por estos autores, como se evidencia en la tabla 1.

Dando lugar a establecer que mejorando parámetros en el reactor de electrocoagulación como: distancia entre electrodos, voltaje, electrolitos de soporte y $\mathrm{pH}$, dicha técnica es la apropiada para reducir los valores de concentración de metales en aguas residuales, cumpliendo con lo permitido por la legislación ambiental. (Acosta et al., 2013).

\section{CONCLUSIONES}

La electrocoagulación es un método atractivo para el tratamiento de diversos tipos de aguas residuales contaminadas con metales pesados, en virtud de los diversos beneficios que incluyen la disminución del impacto ambiental, versatilidad, eficiencia energética y rentabilidad.

Por su parte la industrialización genera altos niveles de contaminación del agua, debido principalmente a los vertidos de aguas residuales, a los efluentes, en muchas ocasiones con una alta concentración de metales pesados, por ende la elección de una técnica de tratamiento de efluentes en un proceso de carácter industrial se rige por diversos parámetros tales como los contaminantes, su concentración, toxicidad, volumen a tratar y toxicidad entre otros, vale la pena recalcar que los esfuerzos que se hagan para la recuperación de aguas residuales no disminuirán según la tendencia observada en esta revisión, ya que esta problemática aún no tiene soluciones eficientes y económicamente viables, seguramente este primer acercamiento a técnicas como la electrocoagulación que han generado datos interesantes tendrá resultados óptimos en los próximos años.

\section{BIBLIOGRAFÍA}

1. Aber, S., Amani-Ghadim, A., \& Mirzajani, V. (2009). Removal of $\mathrm{Cr}(\mathrm{VI})$ from polluted solutions by electrocoagulation: Modeling of experimental results using artificial neural network. Journal of Hazardous Materials, 484-490.

2. Adhoum, N., Monser, L., Bellakhal, N., \& Belgaied, J.-E. (2004). Treatment of electroplating wastewater containing $\mathrm{Cu}^{2+}, \mathrm{Zn}^{2+}$ and $\mathrm{Cr}(\mathrm{VI})$ by electrocoagulation. Journal Hazardous Materials, 207-213.

3. Agouborde, L. (2008). Remoción de metales pesados por medio de adsorbentes no convencionales. Universidad de la frontera. Facultad de Ingeniería, Ciencias y Administración, Temuco, Chile.

4. Acosta, G., Coy, C., Bourdon, A. (2013). Evaluación y regulación de parámetros en un equipo diseñado para la disminución de cromo $(V)$, provenientes de la industria de recubrimientos galvánicos. Ciencia e Ingeniería Neogranadina, 23, 107-116.

5. Akbal, F., \& Camci, S. (2011). Copper, chromium and nickel removal from metal plating wastewater by electrocoagulation. Desalination, 269, 214-222.

6. Arango Ruiz, A., \& Garcés Giraldo, L. F. (2007). Diseño de una celda de electrocoagulación para el tratamiento de aguas residuales de la industria láctea. Universidad Eafit, 56-67.

7. Aristizábal, A., \& Bermúdez, M. (2007). Estudio de la factibilidad de un sistema de electrocoagulación para el tratamiento de aguas procedentes de lavanderias industriales con fines de reuso. Universidad Pontificia Bolivariana, Medellin (Colombia).

8. Arroyo, M., Pérez Herranz, V., Montanés, M., García Antón, J., \& Guinón , J. (2009). Effect of $\mathrm{pH}$ and chloride concentration on the removal of hexavalent chromium in a batch 
electrocoagulation reactor. Journal of Hazardous Materials, 112, 1127-1133.

9. Ashraf, S., Rezayee, M., Mokhtar, A., \& Manouchehr, N. (2010). Removal of Mn 2+ ions from synthetic wastewater by electrocoagulation process. . Desalination, 260, 23-28.

10. Babu, R., Bhadrinarayana, N., Sheriffa Begum, M., \& Anantharaman, N. (2007). Treatment of tannery wastewater by electrocoagulatión. Journal of the University Chemical Technology and metallurgy, 42(2), 201-206.

11. Bahadir, Z., Numa, V., Ozdes, C., Bektas, H., Soylak, M. (2014). Separation and preconcentration of lead, chromium and copper by using with the combination coprecipitationflame atomic absorption spectrometric determination. Journal of Industrial and Engineering Chemistry, 20, 1030-1034.

12. Belkacem, M., Khodir, M., \& Abdelkrim, S. (2010). Using electrocoagulation -electroflotation technology to treat synthetic solution and textile wastewater, two case studies. Desalination, 250, 573-577.

13. Berenguer, A. (2009). Tratamiento de aguas residuales mediante tecnología electroquímica. Universidad de Alicante, España.

14. Bhatti, M., Reddy, A., \& Thukral, A. (2009). Electrocoagulation removal of $\mathrm{Cr}(\mathrm{VI})$ from simulated wastewater using response surface methodology. Journal of Hazardous Materials, 839-846.

15. Chabalina, L. (2002). Propuesta de tecnología para el tratamiento de residuales galvánicos. XVIII Congreso Iberoamericano de ingeniería sanitaria y ambienta. México.

16. Chávez Porras, Á., Cristancho Montenegro, D. L., \& Ospina Granados, É. A. (2009). Una alternativa limpia para el tratamiento de aguas residuales galvánicas: Revisión bibliográfica. Ingenierías Universidad de Medellín, 39-50.

17. Daphia. (2002). Mejora medioambiental del proceso de galvanización. Puesta en marca del protocolo Kioto, 27, 12-15.
18. Domingo, J. (2008). Evaluación de los potenciales riesgos para la salud en el entorno de plantas cementarias: metales pesados, dioxinas y furanos. Jornadas sobre desarrollo sostenible, Barcelona, España.

19. Durante, C., Cuscov, M., Ahmed Isse, A., Sandona, G., \& Gennaro, A. (2011). Advanced oxidation processes coupled with electrocoagulation for the exhaustive abatement of Cr-EDTA. Water research, 2122-2130.

20. Elham, K., Somayeh, Y., \& Mohammad, K. (2011). An investigation on the new operational parameter effective in $\mathrm{Cr}(\mathrm{VI})$ removal efficiency. A study on electrocoagulation by alternating pulse current. Journal of Hazardous Materials, 190, 119-124.

21. Eiband M, TRindade, H., Gama, K., de Melo, mJ., Martinez, C., Ferro, S. (2014). Elimination of $\mathrm{Pb} 2+$ through electrocoagulation Applicability of adsorptive stripping voltammetry for monitoring the lead concentration during its elimination. Journal of Electroanalytical Chemistry, 717-718.

22. Escobar, C., Soto Salazar, C., \& Toral, I. (2006). Optimization of the electrocoagulation process for the removal of copper, lead and cadmium in natural waters and simulated wastewater. Journal Environmental Management, 384-391.

23. Filho, D., Bota, G., Borri, R., \& Cuba, F. (2012). Electrocoagulation/flotation followed by fluidized bed anaerobic reactor applied to tannery effluent treatment. Desalination and Water Treatment, 359-363.

24. FUNDES. (2003). Guía de buenas prácticas para el sector galvanotécnia. Bogotá D.C: Ministerio del Medio Ambiente y FUNDES Colombia.

25. Gilpavas, E. (2008). Aplicación de la electroquímica en el tratamiento de aguas residuales. Universidad EAFIT, 1, 1-85.

26. Golder, A., Samanta, A., \& Ray, S. (2006). Removal of trivalent chromium by electrocoagulation. Separation and Purification Technology, 53(1), 33-41. 
27. Gomes, J., Daida, P., Kesmez, M., Weir, M., Moreno, H., Parga, J., Cocke, D. (2007). Arsenic removal by electrocoagulation using combined Al-Fe electrode system and characterization of products. Journal Hazard Material, 10, 220-231.

28. Gülsün Kılıc, M., Hosten, C., \& Demirci, S. (2009). A parametric comparative study of electrocoagulation and coagulation using ultrafine quartz suspensions. Journal of Hazardous Materials, 247-252.

29. Hagarová, I., Buidos, P., Kubová, J. (2013). Simultaneous preconcentration of cadmium and lead in water samples with silica gel and determination by flame atomic absorption spectrometry. Journal of Environmental Sciences 2013, 25(Suppl.) S45-S49.

30. Heidmann, I., \& Calmano, W. (2008). Removal of $\mathrm{Cr}(\mathrm{VI})$ from model wastewaters by electrocoagulation with Fe electrodes. Separation and Purification Technology, 61,15-21

31. Heidmann, I., \& Calmano, W. (2008). Removal of $\mathrm{Zn}(\mathrm{II}), \mathrm{Cu}(\mathrm{II}), \mathrm{Ni}(\mathrm{II}), \mathrm{Ag}(\mathrm{I})$ and $\mathrm{Cr}(\mathrm{VI})$ present in aqueous solutions by aluminium electrocoagulation. Hazardous Materials, 152, 934-941

32. Holt, P., Barton, G., \& Mitchell, C. (2005). The future for electrocoagulation as a localized water treatment technology. Chemosphere, 355-367.

33. Holt, P., Barton, G., Wark, M., \& Mitchell, C. (2002). A quantitative comparison between chemical dosing and electrocoagulation. Colloids and surfaces, 233-248.

34. Jing-wei, F., Ya-bing, S., Zheng, Z., Ji-biao, Z., Shu, L., \& Yuan-chun, T. (2007). Treatment of tannery wastewater by electrocoagulation. Journal of Environmental Sciences, 19, 1409-1415.

35. Keshmirizadeh, E., Yousefi, S., \& Kazem Rofouei, M. (2011). An investigation on the new operational parameter effective in $\mathrm{Cr}(\mathrm{VI})$ removal efficiency: A study on electrocoagulation by alternating pulse current. Journal of Hazardous Materials, 119-124.
36. Koboya, M., Can, O., \& Mahmut, B. (2003). Treatment of textile wastewaters by electrocoagulation using iron and aluminium electrodes. Journal of Hazardous Materials, 100, 163-178.

37. Linares Hernandez, I., Barrera Díaz, C., Gabriela, R. M., Bilyeu, B., \& Ureña Nuñez, F. (2009). Influence of the anodic material on electrocoagulation performance. Engineering Journal, 148, 97-110.

38. Linares Hernández, I., Barrera Díaz, C., Roa Morales, G., Bilyeu, B., \& Ureña Nuñez, F. (2007). A combined electrocoagulation-sorption process applied to mixed industrial wastewater. Journal of Hazardous Materials, 240-248.

39. Matlock, M., Howerton, B., \& Atwood, D. (2002). Chemical precipitation of lead from lead battery recycling plant wastewater. Chemosphere, 41, 1579-1582.

40. Merzouk, B., Gourich, B., Sekki, A., Madani, K., \& Chibane, M. (2009). Removal turbidity and separation of heavy metals using electrocoagulationelectroflotation technique a case study. Journal of Hazardous Materials, 215-222.

41. Mehtap, G., Hosten, C., Demirci, S. (2009). A parametric comparative study of electrocoagulation and coagulation using ultrafine quartz suspensions. Journal of Hazardous Materials, 247-252.

42. Mólgora, C., Martín, A., Mundo, E., Drogui, P., Buelna, G. 82013). Removal of arsenic from drinking water: A comparative study between electrocoagulation-microfiltration and chemical coagulation-microfiltration processes. Separation and Purification Technology, 118, 645-651.

43. Milanez, K., Kniess, C., Bernardin, H., \& Kuhnen, N. (2005). Caracterização de pigmentos inorgânicos à base de $\mathrm{Fe}$, Zn e Cr utilizando resíduo de galvanoplastia como matéria-prima. Cerâmica. . Sao Paulo.

44. Mollah, M., Morkovsky, P., Gomes, J., Kesmez, M., Parga, J., \& Cocke, D. (2004). Fundamentals, 
present and future perspectives of electrocoagulation. Journal of Hazardous Materials, 114, 199-210.

45. Mollah, Y., Schennach, R., Parga, J., \& Cocke, D. (2001). Electrocoagulation (EC)—science and applications. Journal of Hazardous Materials, 84, 29-41.

46. Morales, B., \& Acosta, G. (2010). Sistema de electrocoagulación como tratamiento de aguas residuales galvánicas. Ciencia e ingeniería Neogranadina, 20, 33-44.

47. Mouedhen, G., Feki, M., G, Petris Wery, M., \& H.F, A. (2008). Behavior of aluminum electrodes in electrocoagulation process. Journal of Hazardous Materials, 150, 124-132.

48. Ölmez, T. (2009). The optimization of $\mathrm{Cr}(\mathrm{VI})$ reduction and removal by electrocoagulation using response surface methodology. Journal of Hazardous Materials, 1371-1378.

49. Patoczka, J., R, J., \& J, S. (1998). The heavy metal removal with ferric chloride. Waste technical conference. Nashville.

50. Ping, G. (2005). Removal of Chromium (VI) from wastewater by combined electrocoagulationelectroflotation without a filter. Separation and purification technology, 43, 117-123.

51. Restrepo Mejia, A. P., Arango Ruiz, A., \& Garcés Giraldo, L. (2006). La Electrocoagulación: retos y oportunidades en el tratamiento de aguas. Corporación Universitaria Lasallista, 1, 1-20.

52. Ritchie, S. (2001). Polycysteine and Other Polyamino Acid Functionalized Microfiltration membranes for heavy metals capture. Enviromental science technology, 35, 3252-3258.

53. Secula, M. S., Cagnon, B., Oliveira, T., Chedeville, O., \& Fauduet, F. (2012). Removal of acid dye from aqueous solutions by electrocoagulation/GAC adsorption coupling: Kinetics and electrical operating costs. Journal of the Taiwan Institute of Chemical Engineers, 767-775.
54. Soto, E., Lozano, T., Barbarin, J., \& Alcala, M. (2004). Remoción de metales pesados en aguas residuales mediante agentes químicos. Ingenierías, VII (23), 46-51.

55. Tchamango, S., Nanseu-Njiki, C., Ngameni, E., Hadjiev, D., \& Darchen, A. (2010). Treatment of dairy effluents by electrocoagulation using aluminium electrodes. Science of the Total Environment, 947-952.

56. Timmes, T., Kim, H.-C., \& Dempsey, B. (2009). Electrocoagulation pretreatment of seawater prior to ultrafiltration: Bench-scale applications for military water purification systems. Desalination, 895-901.

57. Vasudevan, S., Lakshmi, J., \& Ganapathy, S. (2011). Studies on the Al-Zn-In-alloy as anode material for the removal of chromium from drinking water in electrocoagulation process. Desalination, 275, 260-268.

58. Yuan, D., Fu, D., Wang, R., \& Yuan, J. (2008). Rapid determination of chromium(VI) in electroplating waste water by use of a spectrophotometric flow injection system. Spectrochimica acta, 276-279.

59. Zhao, X., Zhang, B., Liu, H., \& Qu, J. (2011). Simultaneous removal of arsenite and fluoride via an integrated electro-oxidation and electrocoagulation process. Chemosphere, 726-729.

60. Zhu, J., Wu, F., Pan, X., Guo, J., \& wen, D. (2011). Removal of antimony from antimony mine flotation wastewater by electrocoagulation with aluminum electrodes. Journal of Environmental Sciences, 23,1066-1071.

61. Zongo, I., Leclerc, J.-P., Maïga, H. A., Wéthé, J., \& Lapicque, F. (2009). Removal of hexavalent chromium from industrial wastewater by electrocoagulation: A comprehensive comparison of aluminium and iron electrodes. Separation and Purification Technology, 159-166. 